\title{
Generic Drug Product Development Bioequivalence Issues
}

Isodore Kanfer, Leon Shargel (Eds).

Drugs and the pharmaceutical sciences, volume 180. Informa Health Care, New York, London, 2008.

This volume is a comprehensive review of the issues in bioequivalence (or perhaps more accurately "therapeutic equivalence") in mid decade. Although most international guidelines concerning bioavailability and bioequivalence (BE) were published in the 1990s, it is hoped that many remaining issues will be resolved in revisions now being considered. Every regulator so involved would benefit from the information in the 12 chapters of this book. While slanted towards the U.S. regulation of generics (or Abbreviated New Drug Applications, ANDAs), aside from semantic differences found in definitions, the scientific issues in $\mathrm{BE}$ discussed are common to all jurisdictions. However, the excellent explanation of the FDA "Orange Book" is mainly for those targeting the US market.

The chapter on bioequivalence of highly variable drugs (HVD $=$ residual $\mathrm{CV}>30 \%$ ) summarises about 15 years of discussions of a very difficult problem and indicates that scaled average BE (ABE), apparently favoured by FDA, may prevail to decrease subject numbers in studies. The chapter on alternate designs and approaches also explores means to accommodate HVD, such as sequential and add-on designs. However, a more thorough examination of the outlier problem in BE studies would have been useful therein. Another chapter reviews population pharmacokinetic studies that are more usually applied in NDAs, but there have been applications for ANDAs in which drugs are ethically administered to patients (e.g., cyclosporine) to prove bioequivalence with sparse sampling. Examples of BE determination are discussed using pharmacodynamic and clinical trial data as applied when BE of inhaled drugs, such as albuterol, are not meaningfully determined with conventional plasma concentration profiles. In those studies, as with HVD, the conventional 90\% confidence interval BE standard of 80 to $125 \%$ may need to be wider to accommodate the high variation exhibited with drug (rather than product) effects.

The chapter on the effect of food spends some time on the NDA drug development concerns, but this is reflective of the FDA "Food effect" guidance. What is not made clear in the chapter is that, when labelling of the reference drug indicates that there is a food effect BE studies for ANDAs require the test vs. reference listed drug to meet the bioequivalence criteria in both fed and fasting states.

Unresolved issues which are reviewed include assessment of endogenous substance BE, implications of chirality and metabolite measurement for BE. In most jurisdictions parent drug, provided it can be measured, is considered to provide profiles that best reflect formulation performance. Overall, this volume is a very useful resource for industry and regulatory agency scientists involved in generic drug product development and assessment.

\section{Iain J. McGilveray}

McGilveray Pharmacon, Ottawa, ON, Canada

mcgilver@magma.ca 